Pesquisa e Reflexão em Educação Básica

\title{
Declive del programa institucional: desafío de la educación juvenil Una mirada desde la experiencia Argentina
}

\begin{abstract}
Resumen:
A partir del declive del programa institucional los docentes deben desarrollar una multiplicidad de trabajos, sobre sí mismos y sobre los otros. Estos otros son tanto estudiantes provenientes de diferentes estratos sociales, producto de la masificación de la escuela secundaria, como con otros docentes, familiares de estudiantes y directivos. Es el sujeto docente quien tiene que en su trabajo, dar coherencia a todo lo que el programa anteriormente daba. Con el declive del programa institucional, ya no hay principios universales válidos, no hay monopolio de la verdad ni de la cultura válida, los sujetos no precisan ingresar a la escuela para conocer el mundo que existe por fuera de sus casas, los medios masivos de comunicación, las redes sociales, la conexión a internet los conectan con distintas noticias, puntos de vista contradictorios, valores distintos.
\end{abstract}

\section{Palabras clave:}

Declive programa institucional. Jóvenes. Educación. Docencia. Argentina.

\section{Declínio do programa institucional: desafio da educação juvenil}

\section{Um olhar a partir da experiência Argentina}

Resumo: A partir do declínio do programa institucional, os professores devem desenvolver uma multiplicidade de trabalhos, sobre si e sobre os outros. Esses outros são alunos de diferentes estratos sociais, produto da superlotação do ensino médio, além de outros professores, familiares de alunos e gestores. É o sujeito docente que em seu trabalho deve dar coerência a tudo o que o programa dava anteriormente. Com o declínio do programa institucional, não há mais princípios universais válidos, não há monopólio da verdade ou cultura válida, os sujeitos não precisam entrar na escola para conhecer o mundo que existe fora de suas casas, os meios de comunicação de massa de comunicação, redes sociais, conexão à internet conectam-nos com notícias diferentes, pontos de vista contraditórios, valores diferentes.

Palavras-chave: Declínio do Programa Institucional. Juventude. Educação. Ensino. Argentina.

1 Licenciada y Profesora en Ciencias de la Comunicación Social, Universidad de Buenos Aires (UBA). E-mail: paula.franco@ yahoo.com.ar. ORDIC iD: https://orcid.org/0000-0002-0551-791X. 


\title{
Decline of the institutional program: challenge of youth education
}

\section{A look from the Argentinian experience}

\begin{abstract}
From the decline of the institutional program, teachers must develop a multiplicity of works, on themselves and on others. These others are both students from different social strata, a product of the overcrowding of secondary school, as well as other teachers, family members of students and managers. It is the teaching subject who has to in his work, give coherence to everything that the program previously gave. With the decline of the institutional program, there are no longer valid universal principles, there is no monopoly of truth or valid culture, subjects do not need to enter school to know the world that exists outside their homes, the mass media of Communication, social networks, the Internet connection connect them with different news, contradictory points of view, different values.
\end{abstract}

Keywords: Institutional Program Decline. Youth. Education. Teaching. Argentina.

\section{Introducción}

El programa institucional no designa un tipo de organización ni un tipo de cultura, sino un modo de socialización, o para ser más precisos, un tipo de relación con el otro (DUBET, 2006). El declive del programa institucional, entendiendo a éste como el proceso que transforma valores en acción y subjetividad, y que, a su vez, designa una manera de llevar a cabo el trabajo sobre los otros, produjo que la escuela deje de ser un santuario, en términos del autor, y sea atravesada por el proceso de la "masificación", el cual ha generado que la escuela secundaria traiga nuevos públicos escolares y se diversifique (DUBET; MARTUCCELLI, 1996).

Estos nuevos públicos que ingresan a la secundaria, se encuentran atravesados por diversas problemáticas socio económicas, cuentan con trayectorias de vida y educativas distintas y, por sobre todo, se trata de sujetos ya constituidos. Eso significa, que no precisan ingresar a la escuela para conocer el mundo que existe por fuera de sus casas: los medios masivos de comunicación, las redes sociales, la conexión a internet, la calle, los conectan con distintos puntos de vistas, y distintos modos de ver la realidad. La escuela así deja de tener el reconocimiento del monopolio de la verdad. Es decir, ésta ya no es considerada como única verdad legítima, ya que se confronta como mínimo con las experiencias y conocimientos de cada sujeto, docente y estudiante, que habita la escuela.

En este marco, el trabajo sobre los otros que realizan los docentes ya no se considera homogéneo, ya no está asegurado por su oficio y rol, sino que se caracteriza por estar atravesado por otra variable que es la personalidad del mismo.

Los docentes ya no realizan un trabajo sobre los otros dirigido a un público homogéneo (en términos sobre todo de trayectorias de clases sociales que ingresan a la escuela secundaria) sino que los sujetos (tanto estudiantes como docentes) se encuentran insertos en un contexto definido, atravesados, en palabras de Bourdieu, por un habitus. Se entiende a éste como el sistema de disposiciones durables y transferibles que implica la forma de percibir el mundo y que, por lo tanto, está ligado a la posición que se ocupa en el mundo social (ÁLVAREZ-URÍA; VARELA, 2009). Así, las trayectorias escolares que configuran los estudiantes no se encuentran alejadas del sistema macrosocial, sino que "[...] se construyen en relación con la cultura, el mercado y la subjetividad de cada individuo" (MÁRQUEZ, 2001, p. 1).

La escuela que durante la vigencia del programa institucional se caracterizaba por su extraterritorialidad, por encontrarse fuera de los "desórdenes mundo", un santuario en palabras de Dubet (2006). Ahora se encuentra atravesada por múltiples factores y heterogeneidades que presentan los estudiantes y docentes. Esto va generando mutaciones en las instituciones tradicionales 
secundarias. Al mismo tiempo, emergen otras con otros formatos y objetivos, como ser educar en diferentes contextos socioeconómicos y zonas geográficas, que generan una dislocación en las funciones que se le asignan a la escuela. En ese sentido la función de distribuciones de certificaciones escolares, a la de producción de un determinado tipo de sujeto y a la de socialización (DUBET; MARTUCCELLI, 1996).

Por otro lado, se considera que el trabajo sobre los otros es una mediación entre los valores universales y los individuos particulares, afirmando que el trabajo de socialización es una vocación porque se funda de valores e involucra normas sobre el individuo. En vigencia del programa institucional el trabajo sobre los otros que realizaban los docentes se definía a partir de la vocación y el rol, en el marco del declive de dicho programa "la distancia entre el trabajo prescrito y el trabajo real se vuelve inevitable [...] por ende es conveniente hablar de experiencia social más que de rol" (DUBET, 2006, p. 91). En la experiencia social va a primar la personalidad, la manera en que cada docente logra darle un sentido a su trabajo y donde el mismo se hace con lo que se es y donde la autenticidad y el compromiso subjetivo se tornan imprescindibles para el desarrollo de la tarea.

Resulta interesante para realizar el presente trabajo y profundizar el análisis y conceptualización, en relación a la situación educativa del nivel secundario en Argentina, teniendo en cuenta que constituye una unidad pedagógica y organizativa destinada a los(as) adolescentes y jóvenes que hayan cumplido con la educación primaria y que abarca un público juvenil que contempla distintos tipos de edades comprendidas entre los 15 y 24 años, en donde se observa como escenario principal la institución educativa en el marco del declive del programa institucional.

\section{Sistema educativo en Argentina}

Las instituciones crean valores y los convierten en emblemas, normas o reglas en pos de brindar metas y acciones sociales para poder regular y apaciguar los conflictos. Así mismo, en las instituciones educativas se juegan procesos de idealización, es decir, "la institución establece ideales y proyectos, y convoca a los individuos a adherir a ellos", (este) "proceso de idealización es vital: compromete el destino de ambas partes" (GARAY, 1994, p. 148).

Actualmente, el sistema educativo argentino está regulado por la Ley de Educación Nacional no 26.206 sancionada en 2006 (ARGENTINA, 2006), que asigna al Estado la responsabilidad de garantizar la igualdad y la gratuidad de la enseñanza. La norma permite que se pueda acceder a una educación de calidad que garantice la igualdad de oportunidades y la equivalencia de los resultados, más allá de las diferencias de origen, según páginas oficiales del gobierno.

El sistema educativo está compuesto de cuatro niveles educativos: inicial, primaria, secundaria y superior, en su mayoría en formato presencial y obligatorio desde los 2 años de educación inicial (a los 4 y 5 años de edad), 6 o 7 años (dependiendo de la jurisdicción) de nivel primario (edad 6 a 11/12 años) y 6 o 5 años (también dependiendo de la jurisdicción) de educación secundaria (13 a 17/18 años). El Ministerio de Educación Nacional solo se encarga de la promoción de la educación y del fomento de las escuelas públicas que sean de carácter nacional.

Según el gobierno nacional, la educación secundaria es obligatoria y constituye una unidad pedagógica y organizativa destinada a los adolescentes y jóvenes que hayan cumplido con la educación primaria. Esta se divide en dos ciclos: un ciclo básico de carácter común a todas las orientaciones y un ciclo orientado de carácter diversificado según distintas áreas del conocimiento, del mundo social y del trabajo.

La autora Nobile (2016), en su artículo académico, considera que la masificación así como la promulgación de la obligatoriedad marcan un cambio en los procesos de desigualdad educativa, ya que si hace décadas atrás la diferenciación se daba principalmente entre quienes asistían o no a la escuela secundaria, actualmente cobran una mayor relevancia las diferenciaciones al interior del 
sistema que conllevan experiencias educativas desiguales (KESSLER, 2002; TIRAMONTI, 2004), y van configurando una "integración excluyente" (BAYÓN, 2015).

El sistema educativo argentino se consolida hacia fines del siglo XIX, y desde esa fecha hasta nuestros días se ha expandido progresivamente en diferentes etapas, que modificaron el rol que ha tenido la escuela secundaria en la sociedad argentina en tanto ámbito establecido como legítimo para la integración social de la población joven. (NOBILE, 2016, p. 4).

Teniendo en cuenta la transformación estructural del país donde se acentúa un proceso de exclusión creciente, se comienzan a construir nuevas subjetividades. Según Fernández (1994) todo establecimiento, para funcionar, reúne una serie de componentes básicos: un espacio material, personas, un currículo, tareas y división de trabajo, y sistemas de organización que regulan las interrelaciones entre las personas y los espacios y elementos materiales. "La interacción de los componentes básicos a lo largo del tiempo arroja como resultado una serie de productos materiales y simbólicos que, en forma genérica, llamamos cultura institucional" (FERNÁNDEZ, 1994, p. 9). Con esa cultura se desenvuelve un estilo específico de relaciones, vínculos, se estructura la toma de decisiones, etc.

\section{¿Qué caracteriza el trabajo sobre los otros?}

Dubet (2013) analiza el trabajo sobre los otros en relación al ejercicio en el campo de la salud, el trabajo social y la educación, ante los objetivos del presente trabajo nos abocaremos a tomar de dicho autor los aportes que nos brinda desde este último campo. Debido al sentimiento de distancia que existe entre el ideal y lo que el mundo es (para lo social) hay un interés de los sujetos para ver cómo responder. Cuando la unidad de la vida social no está dada por la sociedad, por la adecuación entre sistema y la acción, entre una estructura y una cultura, la sociología debe partir del individuo, de su manera de metabolizar lo social y producirlo (DUBET, 2013).

El autor trabaja sobre la categoría de experiencia social y sus mecanismos, las lógicas de acción. Dubet (2013) parte de la premisa de que la experiencia de los sujetos se vuelve cada vez más compleja. Es allí en donde el trabajo de socialización apela a la norma de la conformidad, se percibe la socialización como un proceso activo cuyo verdadero autor debe ser el sujeto.

Dubet escoge el concepto de experiencia social por su doble significado. Por un lado, como "la apertura al mundo, la sensación de ser invadidos e inundados por emociones y sentimientos que nos arrasan y nos superan" (DUBET, 2013, p. 190) y, que más allá de la singularidad de la vivencia tiene su arraigo en lo social y colectivo. Por el otro lado, se contrapone la experiencia como proceso controlado, rigurosamente elaborado y ejecutado.

Aquí radica la potencialidad de la categoría para hacer referencia a la maleabilidad, a la diversidad de lógicas que los actores tienen que llevar adelante en su vida para darle un sentido. Así, las lógicas de acción hacen referencia a los distintos modos que tiene el/la actor/a de relacionarse y abordar sus objetivos. Éstas son: lógicas de integración, por la cual conformamos un identidad colectiva, las lógicas estratégicas, mediante las cuales construye una racionalidad en función de sus objetivos, recursos y posición y, por último, las lógicas de subjetivación, que son aquellas que se dan en la distancia de las otras dos, son aquellas que nos permiten diferenciarnos del resto, tomar decisiones autónomas, aun cuando tengamos nuestros grupos de pertenencia, conformamos un yo que no es idéntico al nosotros.

El trabajo sobre los otros siempre apunta a una integración del prójimo construida sobre un principio de conformidad y de adhesión a los roles propuestos según una forma elemental de socialización (DUBET, 2006). Los jóvenes de hoy en día en las escuelas secundarias están socializados de diferentes maneras, con hábitos, formas, exigencias, saberes pero que deben armonizarse para convivir en la institución a pesar de las tensiones que hay entre ellos en el día a día. 
El trabajo sobre los otros de los docentes se construye y define sobre el oficio (capacidad técnica, autónoma y previsible en el contexto), el rol (posición que se le atribuye en la organización) y la personalidad (capacidad de establecer relaciones que se juzgan conveniente con los sujetos, la cual devendrá de su experiencia profesional). Una de las diferencias entre estas dos últimas es que el rol no pertenece al sujeto y la última, sí (DUBET, 2006).

Durante el programa institucional lo que primaba era el oficio, en tanto los docentes debían cumplir con el estatuto y poner en práctica su conocimiento específico de enseñar para generar un producto claro y objetivable, estudiantes que pasen de grado. Esto se daba a su vez porque el programa garantiza la legitimidad al encontrarse en un rol específico dentro de la organización. El lugar que ocupaban los docentes les confería legitimidad a sus prácticas y saberes, ya que estos no eran puestos en cuestión.

Con el declive del programa institucional, el ingreso del conflicto social a la escuela, la ruptura de su concepción como santuario, también se pone en cuestión la legitimidad y los roles, por lo que ya no están dadas las condiciones para que lxs docentes ejerzan su oficio en el sentido antes mencionado. Es por ello, que cada docente tiene que crear o recrear las condiciones para llevar adelante su trabajo. En principio un trabajo sobre sí mismo, en el cual debe dar sentido y unidad a la multiplicidad de trabajos, lógicas y experiencias diversas y divergentes que lleva adelante y, por otro lado, el trabajo sobre los otros.

En este punto, los docentes implican su personalidad con el fin de conjugar la utilización de distintas lógicas de acción para con los otros y consigo mismo, y darle un sentido a eso. Se desdibuja aquí el oficio, ya que no es un conocimiento, técnica o práctica la que se pone en juego, sino un despliegue de capacidades personales, experiencias que articula. Asimismo el rol en la institución, ya no supone un reconocimiento y es nuevamente la personalidad la que interfiere para crear esa legitimidad a través de distintas estrategias.

Siguiendo a Dubet (2013), los docentes deben imponer y conquistar el oficio en un contexto amenazante, todo converge en la necesidad de dar clases (al igual que los docentes de primaria), pero esas clases no son algo dado, pues la institución no está en condiciones de garantizar su buen desenvolvimiento al recibir estudiantes que no siguen el juego tal como se espera de ellos. Esto también se relaciona con que en el contexto de una sociedad posindustrial, las trayectorias escolares pueden alterarse, discontinuar, finalizar anticipadamente por una infinidad de circunstancias que no estaban contempladas cuando la escolarización era exclusiva de un sector social. Es por ello que se genera un distanciamiento, por un lado, entre las expectativas que docentes y estudiantes tenían de los otros y, por otro lado, de lo que la institución debía garantizar. La pérdida de la reciprocidad de expectativas acentúa la distancia entre el ideal social y las experiencias singulares de los individuos.

Hay una distancia mayor entre las expectativas de los docentes o directivos y los estudiantes, y sus hábitos; tomando a Bourdieu (ÁLVAREZ-URÍA; VARELA, 2009), como el sistema de disposiciones durables y transferibles que implica la forma de percibir el mundo y que, por lo tanto, está ligado a la posición que se ocupa en el mundo social. Este último pone de manifiesto que forma parte de una población para la que no fue pensada la secundaria y ahora tiene que habitarla obligatoriamente.

Teniendo en cuenta el dictado de las clases llevado a cabo por los docentes en las escuelas secundarias de Buenos Aires, es posible considerar que el mismo es característico de un trabajo policentrado (DUBET, 2013), ante la necesidad de innovar en el mismo se “[...] movilizan lógicas heterogéneas convocando al sujeto para que éste cree una unidad y una coherencia que la organización y la técnica ya no le proporcionan"(DUBET, 2006, p. 357). Se pueden vislumbrar lógicas de acción llevadas a cabo por parte de los docentes en la era de la masificación de la escuela secundaria, en relación a las denominadas estratégicas.

El uso del humor, en términos de lógica estratégica, es el medio que suelen utilizar distintos docentes con el objetivo de transmitir los contenidos de la materia que dicta. A su vez, de esa forma, se puede ver 
como la personalidad del docente entra en juego para poder desarrollar su tarea de trabajo sobre los otros y la capacidad que tiene la misma de establecer relaciones que se consideran convenientes y eficaces con los individuos, que deviene de su experiencia profesional, siguiendo a Dubet (2006, p. 353), "se trabaja con lo que se es y la autenticidad y el compromiso subjetivo son esenciales para llevar a cabo (su) actividad".

\section{Condiciones para dar clases - Rol docente}

Las condiciones de educabilidad son las condiciones, recursos, oportunidades para que los alumnos aprendan y los docentes enseñen en diferentes contextos. Las condiciones de educabilidad se refieren al escenario y contexto social y familiar en que se configuran las condiciones socioeconómicas culturales y subjetivas mínimas para concretar la tarea formativa en la escuela. Apunta a identificar cual es el conjunto de recursos, aptitudes o predisposiciones que hacen posible que un niño o adolescente pueda asistir exitosamente a la escuela, al mismo tiempo que invita a analizar cuáles son las condiciones sociales que hacen posible que todos. La noción de condiciones de educabilidad debe ser pensada para posturales estrategias de focalización de igualdad en la diversidad.

Ante la masificación de la escuela el título ya no es signo de distinción, ni la cuna de la única verdad, entonces el por qué estudiar, y cómo motivar para hacerlo es algo que nuevamente, recae en los docentes. Ante esto, los mismos piensan cómo construir las condiciones que permitan dar las clases, pues no alcanza con sólo dar clase para que ésta exista, será necesario articular otras condiciones materiales, y poner en juego algunas estrategias.

Los docentes tienen que desplegar, sin alejarse de su oficio, algunos aspectos específicos de la personalidad. Las dificultades y recursos a utilizar son tan amplios como variables en juego en la diversidad de públicos que irrumpen en la secundaria como por ejemplo: estrategias didácticas novedosas al incorporar el uso de nuevas tecnologías en el dictado de clases para poder llevar a cabo "el cauce de su verdadera actividad: la conversión intelectual de los alumnos" (DUBET, 2006, p. 175).

El docente ya no es el centro de la situación educativa, ya no se encuentra ubicado en un lugar de sujeto supuesto saber. Ante ello emergen prácticas dirigidas al interés de los alumnos y a su motivación. Si en la escuela moderna los sentidos de la escolarización estaban claros, hoy los alumnos pierden el interés por continuar con sus estudios. (AJUN, 2019, p. 306).

Aun cuando Dubet separa como distintas tareas o pruebas a afrontar: la creación de las condiciones para dar clases y la motivación de los estudiantes a hacerlo, se puede relacionar. No sólo porque son dos trabajos extras que se suman a la tarea docente, sino porque la motivación, la voluntad, la movilización de lxs estudiantes en su aprendizaje es una condición indispensable, no para dar clases, pero sí para que aprendan.

En este sentido, el rol de los docentes que se vislumbra más bien de mediador de los relatos, acompañando el proceso de discusión de los temas que se plantean, los docentes se posicionan más como un par, esto enmarcado en que se trata de una organización que se propone la inexistencia de jerarquías entre los conocimientos.

\section{Nuevas formas de educar jóvenes}

Vigotsky (1988) sostiene que la relación que existe entre el sujeto y el objeto cuando se conforma el acto de conocer es una interacción dialéctica, y apunta a una interacción triangular ya que le dará un lugar especial a los artefactos socioculturales que usa el sujeto cuando conoce al objeto. Es determinante, según esta teoría, la intervención del contexto sociocultural. De esta forma, se plantea una ruptura con aquellas teorías que piensan al sujeto de manera individual y biológica. 
La teoría sociohistórica propone pensar el aprendizaje como una práctica social mediada por artefactos y condiciones históricas y culturales. Baquero (2002) retoma determinadas reflexiones de Vigotsky y resalta la importancia del lenguaje en el acto de conocer, ya que lo consideraba un ejemplo canónico de los procesos psicológicos superiores. Por otro lado, sostiene que lo social interviene como un factor inherente a la constitución de los procesos mentales superiores.

"Para que el aprendizaje sea activo, este tiene que implicar experimentar el mundo en formas nuevas" (FRANCO, 2019, p. 9). Es decir, esta postura está lejos de tener una mirada conductista sobre el rol del alumno como sujeto pasivo, con una actividad restringida, etc. Ya no se espera ese modelo de alumno creado por la modernidad con determinadas características ideales, sino que más cercano a la teoría sociohistórica podemos visualizar que los docentes dan cuenta que sus estudiantes son seres sociales, producto y protagonistas en la sociedad, tanto en lo escolar, como en lo extraescolar, incluso teniendo en cuenta la familia y comunidad de cada estudiante. De esa forma, es posible pensar que los estudiantes llegan a la institución con prácticas, conocimientos e ideas previas, lejos de ser tabulas rasas como entiende el conductismo.

Por andamiaje se entiende la interacción entre un sujeto experto, o más experimentado en un dominio, y otro novato, o menos experto, en la que el formato de la interacción tiene por objetivo que el sujeto menos experto se apropie gradualmente del saber experto; el formato debe contemplar que el novato participe desde el comienzo en una tarea reconocidamente compleja, aunque su participación inicial sea sobre aspectos parciales o locales de la actividad global y aun cuando se requiera del "andamiaje" del sujeto más experto para poder resolverse. Por lo tanto, la actividad se resuelve "colaborativamente". El sistema de interacción generado debe promover el desarrollo dentro y más allá de la Zona de Desarrollo Próximo (ZDP) (BAQUERO, 1996).

Asimismo, las estrategias y técnicas de enseñanza se fundamentan en la creación de la ZDP con los alumnos. El docente es el encargado de mediar entre la situación de encuentro entre el alumno y los contenidos socioculturales (HERNÁNDEZ, 1998). Onrubia (2001), señala que, para ello, el docente: A) Insertar actividades que los alumnos realicen en un contexto de objetivos amplios. B) Fomentar la participación e involucramiento de los alumnos en diversas tareas. C) Realizar ajustes continuos de asistencia didáctica. D) Hacer usos del lenguaje para promover la intersubjetividad, entre docentes y alumnos. E) Establecer relaciones explícitas entre los que los alumnos saben, sus conocimientos previos y los que nuevos contenidos de aprendizaje. F) Promover el uso autónomo y regulado de los contenidos. G) Considerar la interacción entre alumnos (HERNÁNDEZ, 1998).

Así, el docente como problematiza el contexto del alumno, y parte de su cotidianeidad, de experiencia previa para diseñar el andamiaje, en un conjunto de actividades que les permitirá alcanzar un buen aprendizaje como resultado de la interacción docente alumno. Cabe recordar que los procesos de apropiación son inherentes a las prácticas de enseñanza. Como indica Baquero (1996) tal asimetría no descansa sólo en el desigual conocimiento que poseen el alumno y maestro, sino también en el desigual poder que poseen para imponer definiciones canónicas de la situación, regular los criterios de trabajo y evaluación.

Repensar formas de aprendizaje sirve para no caer en una visión del saber técnico en donde no se tiene en cuenta un tipo de aprendizaje activo, relacionado a conocer el mundo e incorporarlo, y también un tipo de aprendizaje crítico que incluye el activo y también da lugar a la innovación (FRANCO, 2019).

\section{Conclusiones finales}

El declive del programa institucional implica la ruptura de ideales, y de tomar la institución, los roles, las relaciones como datos objetivos, sino justamente como construcciones sociales que no están determinadas, que para su existencia deben ser constituidas y debe dársele un sentido. Es en este sentido que Dubet (2006, p. 31) plantea que "el orden escolar no es un hecho consumado", y que los docentes deben crear las condiciones para dar una clase, antes de llevarla a cabo. 
A partir de lo desarrollado, es posible concluir que el declive del programa institucional conlleva a que sean los actores quienes tengan que dar sentido a sus prácticas, el trabajar sobre sí mismos para intentar reconstruir una unidad en la propia experiencia que implica lógicas de acción distintas. También reconstruir sino las mismas condiciones que generaba el programa institucional, otras que permitan igualmente desarrollar el objetivo de enseñanza - aprendizaje de la escuela.

La obligatoriedad del nivel en la República Argentina tiene tan sólo 12 años, por lo que la masificación obligatoria es un fenómeno reciente, complicado de aprehender en todas sus aristas. Ya no es sólo cómo crear las condiciones para dar clases, con públicos diversos en clase, habitus, género, nacionalidad, etc, sino también, ¿Cómo construir legitimidad para que haya docentes menores (en relación a la edad) que estudiantes? ¿Cómo romper esa construcción de antaño en que la sabiduría viene de los años/edad? ¿Cómo recuperar esos conocimientos de las experiencias personales (que también son colectivas) de los estudiantes y conjugarlas con los saberes específicos de los docentes?

Sin embargo, es posible entender que la reconstrucción del sentido de la experiencia se intenta crear colectivamente, que ante la falta de principios y valores universales se buscan consensuarlos, que frente a la segregación que provocó la masificación de la escuela secundaria, se busca problematizar y buscar formas de encuentros. Entonces, es posible preguntarse si el fin de la legitimidad instituida e indiscutida de los docentes puede ser el puntapié para lograr un aprendizaje crítico en los estudiantes y a su vez, poder definir de manera colectiva las reglas establecidas en un mismo espacio.

Asimismo las transformaciones pedagógicas promueven el aprendizaje activo, poner al estudiante en el centro, son todas teorizaciones que convergen en el trabajo de los estudiantes como central: para los aprendizajes, para su autonomía, para su dignidad. En ese sentido son pensadas nuevas condiciones de educabilidad en donde se despliegan estrategias y recursos innovadores y novedosos para el público estudiantil.

Es por ello que podemos pensar que el declive del programa puede dar a lugar no sólo a sujetos expuestos a transitar experiencias individuales y reconstruir un sentido, sino también abre la puerta a la creación de espacios más colectivos, más críticos, que deben trabajar para crear las condiciones que ya venían dadas, y en ese trabajo puede discutirse su objeto, sus formas, valores, principios, objetivos.

\section{Referencias}

AJUN, Elyan. Aportes para pensar las prácticas docentes en el declive del programa institucional. El Cardo, Paraná, n. 14, p. 299-312, 2019. Disponible en: http://rct.fcedu.uner.edu.ar/index.php/cardo/article/view/101. Acceso en: 20 enero 2020.

ÁLVAREZ-URÍA, Fernando; VARELA, Julia. Sociología de las instituciones: bases sociales y culturales de la conducta. Madrid: Morata, 2009.

ARGENTINA. Ley n..$^{\circ}$ 26.206, de 14 de diciembre de 2006. Regula el ejercicio del derecho de enseñar y aprender consagrado por el artículo 14 de la Constitución Nacional [...]. Buenos Aires: Congreso de la Nación, [2006].

BAQUERO, Ricardo. La Zona de Desarrollo Próximo y el análisis de las prácticas educativas. In: BAQUERO, Ricardo. Vygotsky y el aprendizaje escolar. Buenos Aires: Aique Grupo Editor S.A., 1996. p. 137-159.

BAQUERO, Ricardo et al. Fracaso escolar, educabilidad y diversidad. In: BAQUERO, Ricardo et al. Ensayos y Experiencias. 1. ed. Buenos Aires: Centro de Publicaciones Educativas y Material Didáctico, 2002. p. 3-11. (Colección Psicología y Educación - no 43).

BAYÓN, María Cristina. La integración excluyente: experiencias, discursos y representaciones de la pobreza urbana en México. México: Bonilla Artigas Editores, 2015.

DUBET, François. El declive de la institución. Barcelona: Gedisa, 2006. 
DUBET François. El trabajo de las sociedades. Buenos Aires: Amorrortu, 2013.

DUBET, François; MARTUCCELLI, Danilo. En la escuela: sociología de la experiencia escolar. Barcelona: Losada, 1996. FERNÁNDEZ, Lidia. Instituciones educativas: dinámicas institucionales en situaciones críticas. Buenos Aires: Paidós, 1994.

FRANCO, Paula Daniela. Jóvenes y entretenimiento ¿es posible aprender? In: JORNADAS DE SOCIOLOGÍA, 13., 2019, Buenos Aires. Actas. Buenos Aires: Facultad de Ciencias Sociales, Universidad de Buenos Aires, 2019. p. 1-16. Mesa 95. Disponible en: https://www.aacademica.org/000-023/564. Acceso en: 20 enero 2020.

GARAY, Lucía. La cuestión institucional de la educación y las escuelas: conceptos y reflexiones. In: BUTELMAN, Ida (org.). Pensando las instituciones: sobre teorías y prácticas en educación. Buenos Aires: Paidós, 1994. p. 227-249.

HERNÁNDEZ ROJAS, Gerardo. Estrategias docentes para un aprendizaje significativo: una interpretación constructivista. México: McGraw-Hill, 1998.

KESSLER, Gabriel. La experiencia escolar fragmentada: Estudiantes y docentes en la escuela media en Buenos Aires. Buenos Aires: Editora IIPE/UNESCO, 2002.

MÁRQUEZ, Francisca. Trayectoria de vida y trabajo en sujetos pobres. Buenos Aires: Editorial Sur, 2001.

NOBILE, Mariana. La escuela secundaria obligatoria en Argentina: Desafíos pendientes para la integración de todos los jóvenes. Ultima década, Santiago, v. 24, n. 44, p. 109-131, jul. 2016. DOI: http://dx.doi.org/10.4067/S0718-22362016000100005. Disponible en: https://scielo.conicyt.cl/scielo.php?script=sci_arttext\&pid=S0718-22362016000100005\&lng=es\&nrm=iso. Acceso en: 20 enero 2020.

ONRUBIA, Javier. Estrategias discursivas y recursos semióticos en la construcción de sistemas de significados compartidos entre profesor y alumnos. Revista investigación en la escuela, Sevilla, n. 45, p. 21-31, 2001.

TIRAMONTI, Guillermina. La fragmentación educativa y los cambios en los factores de estratificación. In: TIRAMONTI, Guillermina (org.). La trama de la desigualdad educativa: mutaciones recientes en la escuela media. Buenos Aires: Manantial, 2004. p. 16-39.

VIGOTSKY, Lev. El desarrollo de los procesos psicológicos superiores. México: Ediciones Crítica Grijalbo, 1988.

Data de submissão: 21/01/2021

Data de aceite: 23/01/2021 
Declive del programa institucional: desafío de la educación juvenil

152 > Cadernos do Aplicação | Porto Alegre | jan.-jun. 2021 | v. 34 | n. 1 